\title{
A two-stage model of visual search
}

\author{
JAMES E. HOFFMAN \\ University of Delaware, Newark, Delaware 19711
}

\begin{abstract}
A two-stage visual search model is described. Four experiments show that in a VM paradigm sequential presentation of the display leads to increases in accuracy and decreases in latency. Slower presentation rates allow for faster transfer of the target from iconic to short-term memory. When target and background items are dissimilar, transfer to short-term memory need not take place and decisions can be made on the basis of the Stage 1 analysis. These results are taken as confirming evidence for the proposed model.
\end{abstract}

At what rate can central brain structures process information arriving over the visual channel? Is the maximum rate limited by the pattern recognition process, memory search, or the necessity to direct attention to a single visual input at a time? It is the thesis of this paper that answers to the above questions lie in viewing the visual information processing system as a hybrid arrangement of parallel and serial processes. This view helps to account for several paradoxical findings in the visual search literature as well as provide a mechanism for the plasticity of search behavior recently revealed by Schneider and Shiffrin (1977).

\section{Sequential Search}

Attempts to directly measure the rate of visual form recognition have used the sequential search paradigm in which an observer attempts to detect the presence of a predefined target character in a sequentially presented visual array. Eriksen and Spencer (1969) found detection accuracy to be independent of presentation rate. Shiffrin and Gardner (1972) replicated this finding for a case in which the spatiotemporal order of inputs was known in advance by the observer. Eriksen and Spencer (1969) and Kinchla (1974) proposed that each display character was recognized in parallel and independently (the independent channels, or IC, model). Further refinements of the IC model were provided by Estes (1972), Gardner (1973), and Shiffrin and Geisler (1973).

Schneider and Shiffrin (1977) proposed that when the subject searches for the same character set consistently (consistent mapping, or CM), the members of that set are eventually recognized through a process of "automatic detection" whose operating time is independent of the number of characters in the dis-

This research was supported by NIMH Grant 27110 . The author thanks Carolyn Johnson for helpful comments on an earlier draft of this paper. Requests for reprints should be addressed to the author at the Department of Psychology, University of Delaware, Newark, Delaware 19711. play or the target set. When the target set periodically switches roles with the background set (varied mapping, or VM), automatic detection is not possible and the subject searches the array by comparing each member of the target set to each item in the display, terminating when a match is located.

The distinction between two different search modes and the emphasis on the subjects' experience helped resolve many of the conflicting findings of different search experiments. Nonetheless, there are several findings that do not appear to be compatible with either of the two search modes.

Sternberg and Scarborough (1969) had subjects search a sequentially presented array for any one of five characters. Letters were presented at a rate of $1 / 75$ msec. It appeared that targets were located by a serial exhaustive comparison to the memory set. In the case of a five-letter memory set, this process would require $200 \mathrm{msec}$. If each letter in the display were being evaluated by such a process, then the fast presentation rate would require that several letters be simultaneously present in the comparison stage. Thus, the memory comparison process does not set a limit on the rate of search.

A related finding is that of Shaw (1977), who had subjects search a linear string of letters in a prescribed order. RT was an additive combination of memory set size and the position of the target in the string. In agreement with Sternberg and Scarborough (1969), search rate was independent of memory set size.

Hoffman (1978) extended the Eriksen and Spencer (1969) paradigm using a VM procedure. The display format is shown in Figure 1 (also the format used in the experiments to be reported here). The threeletter sets shown in Figure 1 periodically exchanged roles as target and background sets. Detection accuracy increased as the interval between characters (stimulus onset asynchrony, or SOA) increased, contrary to the predictions of the IC model.

Latency data, however, indicated that the departure from the predictions of the IC model could not be attributed to a serial comparison process such as proposed by Schneider and Shiffrin (1977). A high- 


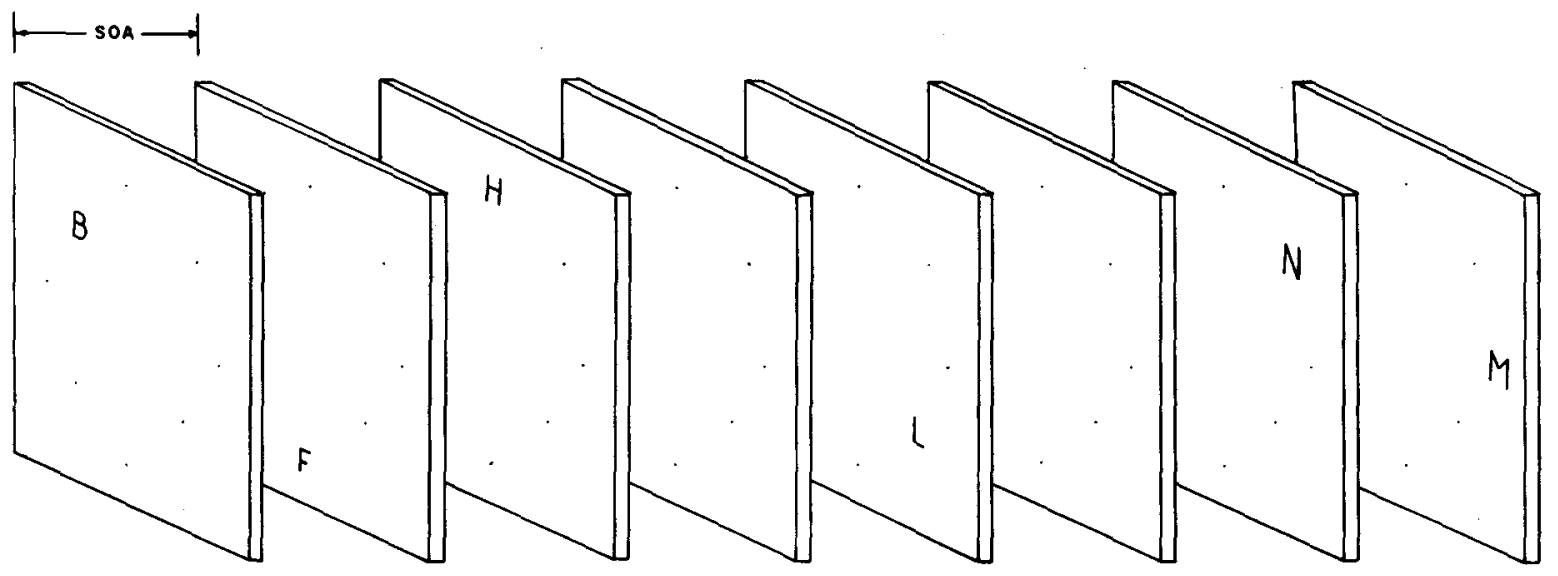

MEMORY SET: PVDH

BACKGROUND SET: RBNF LXMG

Figure 1. The sequential search paradigm. Each display letter appears in a different time frame and spatial location.

accuracy sequential search paradigm showed a curious pattern of results. Decreasing presentation rate produced a small decrease in positive latencies (target present) and a much larger one on negative trials (target absent). The decrease on positive trials was too small to be attributed to a serial search. In addition, the SOA at which latencies no longer continued to decline was independent of memory set size. Given that this SOA value represents the time required to classify a character before the next one arrives, a paradox is apparent. Although increasing memory set size produces a linear increase in RT, it does not set the limit on character classification time. This finding is very similar to those of Shaw (1977) and Sternberg and Scarborough (1969), reviewed above, which indicate that the memory comparison process is not the limiting factor in rate of visual search.

Hoffman (1978) proposed that subjects can search a rapidly presented display using a slow memory comparison process because display elements are first evaluated by a parallel process. Only those forms which bear a physical resemblance to the memory set are admitted to the serial comparison process. A computer simulation of this two-stage model provided a good quantitative account of accuracy and latency in the sequential presentation paradigm. A description of this model follows.

\section{A VISUAL SEARCH MODEL}

Several years ago, Neisser (1967) outlined a twostage theory of visual search. An initial "preattentive" stage was responsible for segmentation of the scene and was parallel, although error prone. It guided a second stage of focal attention which performed fine discriminations on a single object at a time. The model we present is closely related to Neisser's theory.

In outline, the model works as follows. Each item in the display is compared in parallel to all memory set items. The outcome is a similarity measure for each display item. Display characters are then serially transferred, in order of decreasing similarity, to a serial exhaustive comparison process. The transfer mechanism is assumed to be the same spatial selective attention mechanism studied in detail by Colegate, Hoffman, and Eriksen (1973) and Hoffman (1975). A detailed description follows.

\section{Stage 1}

Anderson (1973), Kinchla (1974), and Lappin (Note 1) have described parallel signal processing mechanisms, based on statistical communication theory, which accomplish signal detection by passing the signal through a filter. When one of several signals must be detected, the outputs of several filters are integrated, which increases the noise in the detection process and lowers the signal/noise ratio. This idea forms the basis for an analogue to "preattentive processes" (Neisser, 1967). Assume that each memory set item can be represented as a filter and that the outputs of the filters are integrated. Increasing memory set size will lead to more noise in the output and a lower signal/noise ratio.

In particular, let

$$
S_{i}=\sum_{j=1}^{M} X_{j},
$$

where $S_{i}$ is the similarity value for the $i^{\text {th }}$ display element and $M$ is the size of the memory set. $X_{j}$ is 
a normally distributed random variable with expected value and variance as follows:

$$
\begin{aligned}
E\left(x_{j}\right)= & 0 \text { for a noise element } \\
= & \mu \text { for a target element } \\
& \operatorname{VAR}\left(X_{j}\right)=\sigma^{2} .
\end{aligned}
$$

Then, if the subject has $\mathbf{M}$ letters in his memory set, the expected value and variance of the similarity value, $S_{i}$, are as follows:

$$
\begin{gathered}
\mathrm{E}\left(\mathrm{S}_{\mathrm{i}}\right)=0 \text { for noise elements } \\
=\mu \text { for a target element } \\
\operatorname{VAR}\left(\mathrm{S}_{\mathrm{i}}\right)=\mathbf{M} \sigma^{2} .
\end{gathered}
$$

Thus, the signal/noise ratio, analogous to the $d^{\prime}$ measure of signal detection theory is

$$
\text { S/No }=\mu / \sqrt{\mathrm{M \sigma}^{2}} \text {. }
$$

In other words, the signal-to-noise ratio of the similarity value goes down with increasing memory set size.

The subject could, at this point, make a decision. The optimal procedure is to sum the similarity values corresponding to each display element and compare the sum to a criterion. Kinchla (1974) described this model and showed that it fit the Eriksen and Spencer (1969) data as well as his own data on redundant targets. Thus, a subject responding on the basis of Stage 1 analysis will behave as CM subjects do.

When the signal/noise ratio is low, as might happen in VM circumstances or when signal and noise items are similar, responding on the basis of Stage 1 would produce a high error rate. This error rate could be reduced by submitting items to a second, slower, more accurate discrimination process. We assumed that a candidate list was formed by comparing each similarity value to a criterion. All those items passing the criterion were placed on the candidate list. Items not placed on the candidate list received no further processing.

\section{Transfer}

Candidates are transferred to Stage 2 processing by a process of selective attention. That item currently having the highest similarity value is transferred first. Transfer time is a random variable having a rectangular distribution on the interval $0-200 \mathrm{msec}$. This assumption fits reasonably well with the data on spatial selective attention cited earlier. Once trans- fer is complete, transfer of another element may begin (assuming another candidate currently exists). Thus, the transfer of or attention to display items can be conducted in parallel with memory comparison. However, if a display item completes transfer while a preceding one is still undergoing Stage 2 processing, the second one must queue. Due to this arrangement, the asymptotic SOA value will depend on the slower of two processes: the attentional mechanism or Stage 2 comparison. In many experiments, the duration of the attention mechanism is longest leading to an asymptotic SOA value that is not dependent on memory set size.

Notice that if the signal/noise ratio is high, the signal will, in general, be the first item transferred and there will be very little effect of rate on positive trials. On negative trials, however, the entire list must be examined, leading to large savings in RT with sequential presentation.

\section{Stage 2}

Stage 2 is a serial exhaustive comparison process. Only one item at a time can be present in this stage. At the end of an exhaustive memory comparison, if a match has been found, a positive response is initiated, otherwise a new display item is entered until either all comparisons have been made or limitations of iconic memory prevent any further comparisons. Stage 2 is assumed to be error free. Thus, the main sources of errors (misses) are the failure of signal items to gain admission to the candidate list and the elimination of items from the candidate list when their waiting time exceeds iconic memory duration. False alarms are assumed to be guesses.

The present series of experiments was designed to test several implications of the two-stage search model. The model suggests that increasing detection accuracy with decreasing presentation rate in VM paradigms is due to a process limitation caused by a serial transfer of the display items to short-term memory for Stage 2 processing. A failure to find rate effects is attributable to the subject's relying on the Stage 1 analysis.

Since the effect of rate on detection accuracy is of crucial importance in rejecting the IC model, it is desirable to verify that the effects of rate are not due to data limitations.

Previous experiments showing increased accuracy with slower presentation rates (Schneider \& Shiffrin, 1977; Sperling, Budiansky, Spivak, \& Johnson, 1971) have presented the sequential characters to the same retinal locations and have left the display on during the interstimulus interval (ISI). Detection accuracy under these conditions would improve with increases in the ISI because of decreased forward and backward masking as well as increases in the energy of the display due to temporal integration. Both studies 

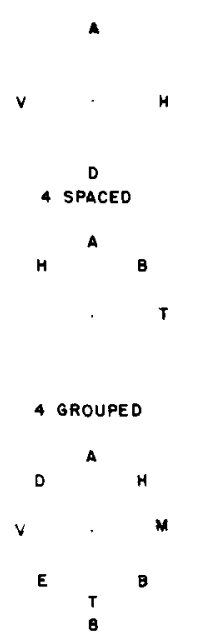

Figure 2. Three display formats utilized in Experiment 1. The four-grouped and eight-letter displays share the same intercharacter spacing.

mentioned above found an effect of rate even in a $\mathrm{CM}$ paradigm where the subject should not be sensitive to processing load. The paradigm illustrated in Figure 1 avoids this limitation. However, one possible data limitation in Hoffman's (1978) experiments is lateral masking between adjacent display characters. Sequential presentation would reduce this masking and lead to increase in detection accuracy. Experiment 1 evaluated this possibility using the three display formats shown in Figure 2. If the effect of rate on detection accuracy is to reduce lateral masking, then we would expect the four-grouped and eight-letter displays to show similar effects of the rate variable since they have the same intercharacter spacing. ${ }^{1}$ The four-spaced condition, in which the elements are separated by a distance greater than that over which contour interference takes place, should show no effect of presentation rate.

\section{EXPERIMENT 1}

\section{Method}

Subjects. The author and five paid volunteers served as subjects.

Apparatus and Stimuli. The letters were $.23^{\circ}$ high and constructed by illuminating the appropriate points in a 6 by 5 matrix on a Tektronix 604 display monitor equipped with $P-15$ phosphor under control of a PDP-12 computer. Each point had a luminous directional intensity of $2.8 \times 10^{-7} \mathrm{~cd}$ on a dark background. Letters appeared on the circumference of an imaginary circle, $2.1^{\circ}$ in diameter and centered on a fixation point.

Procedure. Each subject served in one practice and two experimental sessions. Each session consisted of six blocks of trials, one block for each combination of three memory set sizes (one, two, or four letters), and two rates ( 0 and $75 \mathrm{msec})$. Each block consisted of 192 trials, half of which were positive. The order of display types (four grouped, spaced, and eight letters) within the block was random within the constraint that each member of each memory set occurred within each display type and each temporal position equally often. Targets occurred in each of the eight possible temporal positions equally often.
The composition of the memory set changed within blocks as follows. The letters comprised three sets (AXPL, HMBT, and DUEI). Let $\mathrm{N}$ be the size of the memory set. The first $\mathrm{N}$ members of Set 1 served as the memory set for $\mathrm{N} \times 24$ trials. Noise elements were chosen from Sets 2 and 3 . Following this, the next $\mathbf{N}$ members of Set 1 became the memory set until Set 1 was exhausted, at which time, Set 2 became the memory set with noise elements chosen from Sets 1 and 3 . Set 3 was not used for memory set elements. The advantages of this procedure are that the subject sees essentially identical displays independent of memory set size, and the members of the sets are well learned.

On each trial, the subject fixated a light point and initiated a trial with a microswitch. Four or eight letters appeared sequentially around the circumference of an imaginary circle. The spatial ordering of letters was random, as was the spatial position of the target on positive trials. Positive latencies were measured from onset of the target element, while negative latencies were measured from onset of the last item in the sequence. Displays were not refreshed.

The subject pressed one of two microswitches to indicate whether or not he detected a match between any display element and memory set item, and received immediate feedback on correctness of response and latency.

\section{Results and Discussion}

Figure 3 shows hit rate and false alarm rate as a function of memory set size, SOA (0 or $75 \mathrm{msec}$ ) and display type. Each point is based on approximately 384 observations. The data for the eight-letter display condition replicate those reported by Hoffman (1978) in showing decreased detection accuracy with increased memory set size and presentation rate. The data for the four-letter displays indicate that the gain in accuracy with decreased rate is indeed a process limitation. The two four-letter conditions (spaced and grouped) are virtually identical and show little sensitivity to variation in rate. We thus conclude that the gain in accuracy with decreased rate reported in Hoffman (1978) and replicated here is due to a limitation in the ability to process several simultaneous inputs when the subject is in a VM paradigm.

Hit rate and false alarm rate were used to compute a d' measure assuming equal variance, normally distributed noise, and signal-plus-noise events. Repeated-measures analysis of variance on these scores revealed significant effects of rate $[F(1,5)=8.5$, $p<.05]$, display type $[F(2,10)=32.3, p<.001]$,
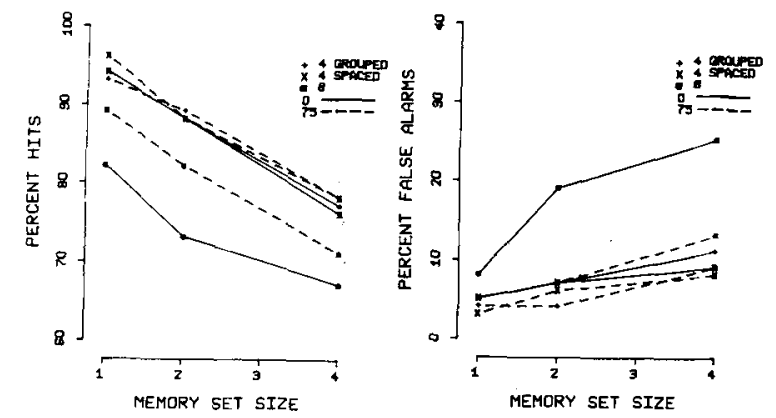

Figure 3. Hit rate and false alarm rate as a function of memory set size, presentation rate, and display format. Experiment 1. 
memory set size $[F(2,10)=56.2, p<.001]$, and the Rate by Display Type interaction $[\mathrm{F}(2,10)=14.2$, $\mathrm{p}<.001]$. All other interactions were nonsignificant.

Figure 4 shows average correct reaction time as a function of memory set size, display type, and SOA for positive and negative responses. RT is approximately a linear function of memory set size as Hoffman (1978) and Sternberg and Scarborough (1969) reported. Our subjects produced an error rate of about $18 \%$ with a four-letter memory set, eightletter display, and an SOA of $75 \mathrm{msec}$, compared to the $10 \%$ Sternberg and Scarborough (1969) report for a five-letter memory set. The error rate here would be much larger if the subjects skipped those items arriving while the comparison process was occupied.

The effect of rate on $\mathrm{RT}$ is virtually identical to that reported by Hoffman (1978) for an eight-letter display. On positive trials, there is a very small savings in RT with sequential presentation, while on negative trials there is a sizable reduction. The reason for such a small savings in $\mathrm{RT}$ on positive trials is that search through the display is not random; it is guided by an initial analysis which ensures that the target item will usually be the first one transferred. The effect of rate, and the cause of errors, is attributable to those trials on which the target must queue. A very similar pattern of RT results has been obtained in an experiment in which accuracy is virtually perfect (Hoffman, 1978).

The results of Figure 4 also confirm our conclusions regarding the effect of display type. The results for the two four-letter displays are identical. Together with the accuracy results, this indicates that the primary variable determining our results is the number of inputs and not their spacing.

One anomalous finding is the faster RT for an eight-letter display, 75-msec SOA on negative trials relative to four-letter displays. It is not obvious what information subjects used to guide their negative
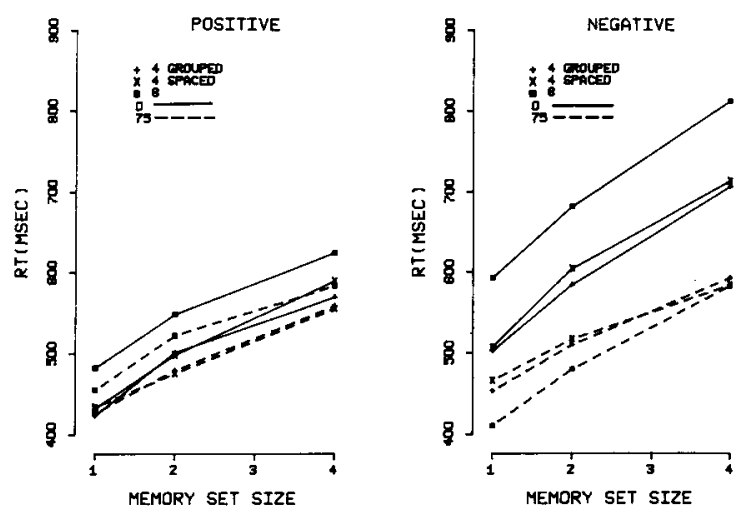

Figure 4. Average correct latency as a function of memory set size, presentation rate, and display format for positive and negative trials. Experiment 1. responses-whether it was an implicit counting of inputs, estimated time, or elapsed time since last input. Since the three display types occurred randomly in a block, there was more uncertainty at the end of four inputs as opposed to eight that the entire display had been presented. This is undoubtedly the cause of this puzzling finding.

A repeated-measures analysis of variance revealed all main effects and their interactions to be significant $(\mathrm{p}<.05)$ except for the following: Display by Response Type (positive or negative), Display Type by Memory Set Size, Rate by Display Type by Memory Set Size, and Rate by Display Type by Memory Set Size by Response Type.

Sternberg and Scarborough (1969) suggested that one way a slow comparison stage could accurately process a fast sequence of inputs would be to queue any letters arriving while a comparison was taking place. They reported that RT was independent of the temporal position of the target in the input sequence, contrary to the queuing hypothesis. We examined the effect of temporal position, grouped into four pairs- $(1,2)(3,4)(5,6)(7,8)$-on search accuracy. Temporal position was found to have a highly significant effect on percent hits $[F(3,15)=$ $13.4, \mathrm{p}<.0011$. The subjects were less accurate on later temporal positions. This effect did not depend on the duration of the memory comparison process, since the Temporal Position by Memory Set Size interaction was not significant $[\mathrm{F}(6,30)=1.0$, $\mathrm{p}<.4]$.

RT was also significantly affected by temporal position $[\mathrm{F}(3,15)=11.1, \mathrm{p}<.001]$. Here, temporal position interacted with memory set size $[\mathrm{F}(6,30)=$ $5.8, \mathrm{p}<.001]$ but not in the way to be expected on the basis of the queuing theory. Although RT was slower for later temporal positions, the effect was larger for smaller memory set sizes.

The reason for the discrepancy with the results of Sternberg and Scarborough (1969) is the requirement in this experiment that the subjects make an explicit negative response. As successive letters are examined without finding a target, the probability of a target's occurring is decreasing. Thus, as the end of the sequence approaches, the subjects have a high criterion for a positive decision with an attendant increase in positive $\mathrm{RT}$ and misses. This trend can be reversed by simply concentrating the occurrence of targets in the last two temporal positions (Hoffman, 1978).

In summary, the results of Experiment 1 indicate that subjects benefit from sequential presentation of a display in a VM paradigm. This benefit reflects a process limitation and cannot be attributed to confounding effects of backward, forward, or lateral masking. In agreement with Sternberg and Scarborough (1969), RT was dependent on memory set size, suggesting a serial comparison process. 
According to the two-stage search model, the process limitation affected by presentation rate is the ability to transfer items from a rapidly fading trace (iconic memory) to a more permanent store (STM). Sequential presentation allows the target to be transferred sooner, reflected in the small RT savings on positive trials. The second experiment was designed to test this conjecture, using visual masking. If the target is being transferred from a visual representation to STM faster with sequential presentation, then the target should be able to evade an aftercoming visual noise mask sooner under sequential presentation conditions. We presented the subjects with simultaneous (SOA $=0 \mathrm{msec}$ ) or sequentially (SOA = $75 \mathrm{msec}$ ) presented arrays in which each display character was followed by a pattern mask at various delays. We predicted that the masking function would reach a no-mask control level of accuracy at shorter delays for a sequentially presented array as opposed to a simultaneous array.

\section{EXPERIMENT 2}

\section{Method}

Subjects. The subjects were the author and three volunteers who were paid for their services.

Apparatus and Stimuli. All details of apparatus and stimuli were identical to those of Experiment 1, with the following exceptions: characters appeared on the face of a Digital Equipment Corp. VR-14 oscilloscope equipped with P-31 phosphor. The stimuli were MDTJ, LHFV, and AXRP. Characters were followed by a pattern mask, "\$."

Procedure. Each subject served in one practice and four experimental sessions. Each session consisted of six blocks of 192 trials, each block devoted to one of six mask interstimulus intervals (MISI) of 50,100,200,250, and $300 \mathrm{msec}$ and a no-mask control condition (NM). The same nested memory set procedure was employed as in Experiment 1. Memory set size was fixed at four. The order of conditions within a session was random.

Each session was devoted to one of two presentation rates: 0 - or $75-\mathrm{msec}$ SOA. These values alternated over sessions with the starting value counterbalanced over subjects.

- On initiating a trial, a subject was presented with a letter display presented at 0 - or $75-\mathrm{msec}$ SOA. Each character in the array was followed by a pattern mask at one of the six MISI values. All other procedural details are identical to those of Experiment 1.

\section{Results}

The hit rate and false alarm rate data were used to compute $d$ ' values assuming equal variance, normally distributed noise, and signal-plus-noise events. These values are shown in Figure 5. Each point is based on approximately 1,636 observations. It is clear that we replicate the improvement in accuracy with slower presentation rate obtained in Experiment $1[\mathrm{~F}(1,3)$ $=35.0, \mathrm{p}<.001]$. There is also the common monotonic improvement in accuracy with increasing delays of the mask $[\mathrm{F}(5,15)=55.6, \mathrm{p}<.001]$. The interaction of MISI with rate is not significant $[F(5,15)$ $=1.1, \mathrm{p}<.3]$.

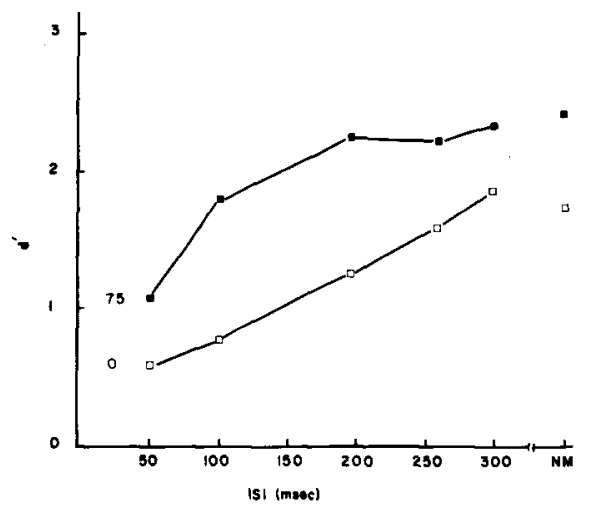

Figure 5. $d^{\prime}$ as a function of the interval between each display character and a subsequent pattern mask for displays presented at two different rates. Experiment 2.

A Dunnett test (Winer, 1962, pp. 90-91) for comparing treatment conditions with a control was conducted separately on the two presentation rate conditions using a criterion of $\mathrm{p}<.05$. For the $0-\mathrm{msec}$ presentation rate data, accuracy was significantly different from no-mask control performance at all MISI values less than $250 \mathrm{msec}$. The corresponding value for the 75-msec SOA data was $200 \mathrm{msec}$.

Thus, it appears that our conjecture that a target letter in a sequentially presented display will evade an aftercoming mask sooner than if it is embedded in a simultaneous array is confirmed. This conclusion should be viewed with caution, however, since the effect is small (as is the reduction in RT on positive trials with sequential presentation) and it is based on examining the shapes of functions at two different absolute levels of performance. Spencer (1971) did not find any difference in masking ranges for equal energy conditions, such as those used here, and equal performance conditions. Nonetheless, we only view this finding as suggestive that the effect of presentation rate is operative at a stage when the display representation is still visual in nature.

Another aspect of the data in Figure 5 is interesting. There is little evidence here that the subject switches to each new letter as it arrives in the sequential condition, as Schneider and Shiffrin (1977) proposed for their paradigm. If the subject adopted such a switching strategy, an aftercoming mask would not affect performance once it was delayed beyond $75 \mathrm{msec}$. However, it is clear that a mask is still effective beyond this delay, suggesting that processing of a letter is proceeding as other letters are arriving in iconic store.

The first two experiments together reveal that a subject in a VM paradigm is limited in his ability to process several simultaneous inputs. This limitation is not due to lateral interference from adjacent letters or from backward or forward masking produced by sequential presentation. It appears that the 
limitation occurs because the subject must serially transfer display elements from a visual store to a more permanent memory where a serial comparison may occur.

According to the two-stage model proposed by Hoffman (1978), the transfer of items from iconic to short-term memory is not random but is guided by an initial parallel evaluation. If the signal/noise ratio is high, the target item will be the first element transferred, leading to small effects of presentation rate on positive trials. If the signal/noise ratio is high enough, the subject may rely completely on the Stage 1 analysis and show no effect of rate or memory set size.

Experiments 3 and 4 were designed to test these ideas. In these experiments, the subject searched for a target embedded in two types of context: the letter context utilized in the first two experiments and a symbol context in which nontarget locations were occupied by the character " $\$$." According to the two-stage model, decreasing presentation rate will produce a savings in RT because occasionally noise items will generate a higher Stage 1 similarity value than the target. On these trials, the target will have to queue to gain access to the serial exhaustive comparison stage and a savings in RT will result with sequential presentation. If the signal/noise ratio is high, in this case because the symbol context bears little similarity to the letters, the single letter in the display should always be transferred first and no advantage of sequential presentation should result. Thus, in Experiment 3, RT in the symbol context should not be sensitive to variation in rate, but should reveal an effect of memory set size since Stage 2 analysis would be required to discriminate whether the letter was from the positive or negative set.

Experiment 4 differed only in using the symbol context to fill up the array on negative trials. If the subject can discriminate at Stage 1 between letters and symbols, as we implied above, then he should be able to skip Stage 2, thereby appearing to be a parallel processor. RT should show no effect of rate and, in addition, no effect of memory set size.

\section{EXPERIMENTS 3 AND 4}

\section{Method}

Subjects. The author and three paid volunteers served as subjects.

Apparatus and Stimuli. Apparatus and stimuli were identical to the previous experiment, with the exception of the use of " $\$$ " as a display character.

Procedure. Each subject served in one practice and two experimental sessions, one session devoted to the letter or symbol context. The order of sessions was counterbalanced across subjects. In the symbol context, a single letter appeared in the display, other positions being occupied by the " $\$$ " character. On negative trials, the letter was randomly chosen from the set of eight pos-
Table 1

Percent Correct as a Function of Memory Set Size, Presentation Rate, and Context

\begin{tabular}{lrccc} 
& \multicolumn{4}{c}{ Memory Set Size } \\
\cline { 2 - 5 } Context & & 1 & 2 & 4 \\
\hline \multirow{2}{*}{ Symbols } & 0 & 97 & 97 & 96 \\
& 75 & 98 & 97 & 96 \\
Letters & 0 & 95 & 90 & 86 \\
& 75 & 96 & 92 & 93 \\
\hline
\end{tabular}

sible nonmemory set characters. The target letter could occur in Temporal Positions 2, 4, or 6. Other details of procedure were identical to the previous experiment. Experiment 4 used the same procedure, except that in the symbol context on negative trials, the display was a homogeneous set of " $\$$ " characters.

\section{Results and Discussion}

Accuracy was indexed by percent correct, since performance was perfect for some conditions for each subject, leaving $\mathrm{d}^{\prime}$ undefined. Table 1 shows percent correct averaged over subjects. Results for the letter context replicate the previous two experiments in showing increased accuracy with smaller memory sets and slower presentation rates. The effect is smaller here, probably due to the degree of practice these subjects received through the sequence of experiments. Accuracy in the symbol context is uniformly high and shows little effect of the various independent variables.

A repeated-measures analysis of variance on these scores shows a significant effect of context $[F(1,3)=$ $11.9, \mathrm{p}<.05]$, rate $[\mathrm{F}(1,3)=30.9, \mathrm{p}<.001]$, memory set size $[F(2,6)=8.9, p<.02]$, and the Context by Rate interaction $[\mathrm{F}(1,3)=17.0, \mathrm{p}<.05]$.

Correct RT averaged over subjects and trials is shown in Figure 6. The results for the letter context replicate those found in Experiment 1 . We predicted that rate would have differential effects on the two
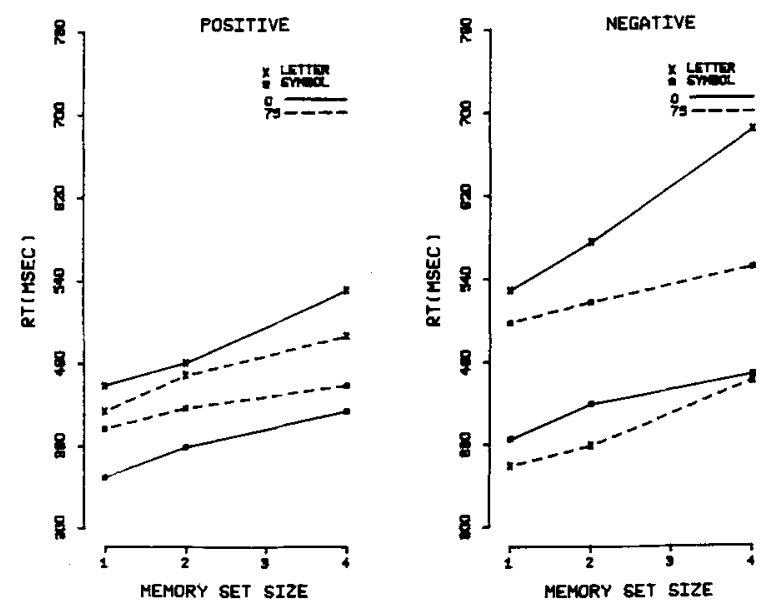

Figure 6. Average correct latency as a function of memory set size, presentation rate, and context (letters or symbols) for positive and negative trials. Expriment 3. 
context conditions. This prediction is supported, but in a way that was not anticipated. These results show that RT is actually faster in the symbol context for the 0 -msec SOA condition than the $75-\mathrm{msec}$ rate. This finding is not predicted by the two-stage model, and suggests that a revision is in order. In addition to the Stage 1 analysis, which involves memory comparison, there must be a prior, parallel stage which is sensitive to "global" features of the pattern, those features not depending on memory comparison. This stage is more efficient with more simultaneous identical patterns. Uttal (1975) proposed an autocorrelation detector that would display many of these characteristics. Lappin and Bell (1975) implicated a similar mechanism (cross correlation) responsible for detecting movement between sequentially presented random dot patterns. These various proposals all emphasize a pattern-detection mechanism that may be sensitive to periodicity in space and time. The sequential presentation technique offers a useful method of isolating configurational factors in visual search, for they are disrupted by temporal discontinuity.

A repeated-measures analysis of variance revealed significant effects of all variables and their interactions, except for the following: rate, Context by Response Type, and Rate by Response Type. All three- and four-way interactions were not significant. Of primary interest were the effects of context $[\mathrm{F}(1,3)=663.4, \mathrm{p}<.001]$, Context by Rate $[\mathrm{F}(1,3)$ $=34.9, \mathrm{p}<.001]$, and Context by Memory Set Size $[\mathrm{F}(2,6)=24.4, \mathrm{p}<.001]$.

The accuracy data for Experiment 4 was similar in all respects to that of Experiment 3 and will not be further considered. Correct RT for Experiment 4 is shown in Figure 7. We again obtained the now familiar effects of rate, memory set size, and re-
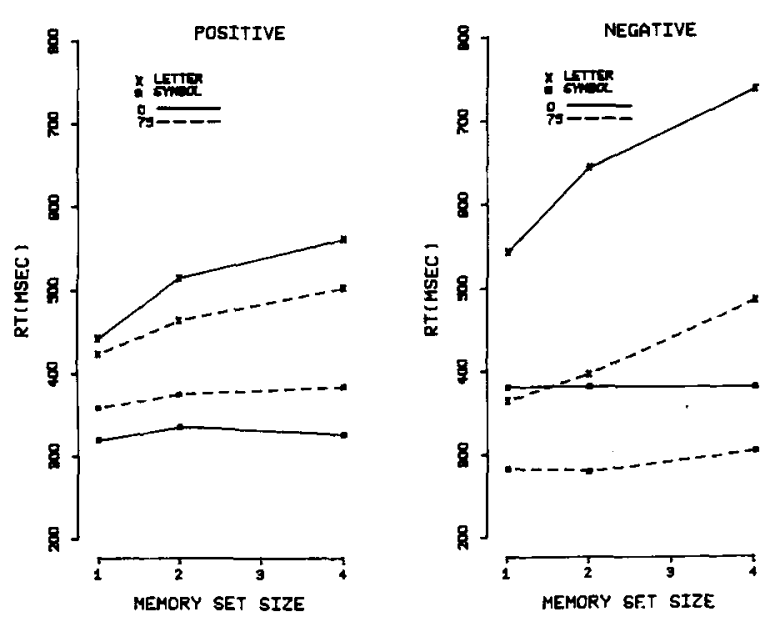

Figure 7. Average correct latency as a function of memory set size, presentation rate, and context (letters or symbols) for positive and negative trials. Experiment 4. sponse type for the letter context. However, the symbol context shows a dramatic departure from previous results. In agreement with the previous experiment, RT is slower with sequential presentation than with simultaneous presentation. In contrast with previous experiments, however, the effect of memory set size has been eliminated. Memory comparison is not required when a prior parallel analysis can efficiently provide information on the presence or absence of a target.

A repeated-measures analysis of variance on correct $\mathrm{RT}$ revealed significant effects of all factors and interactions $(p<.05)$ except for the following: response type, Context by Response Type, Context by Rate by Response Type, and Rate by Response Type by Memory Set Size. Of primary interest were the effects of context $[F(1,3)=89.9, p<.001]$, Context by Memory Set Size $[\mathrm{F}(2,6)=59.9, \mathrm{p}<.001]$, and Context by Rate $[F(1,3)=22.3, p<.001]$.

Experiments 3 and 4 together provide strong evidence for different stages operating in visual search and constitute qualitative support for the two-stage visual search model outlined earlier.

\section{GENERAL DISCUSSION}

The visual search literature presents several paradoxical findings. Rate of search is very rapid relative to the time required for memory comparison and spatial selection of individual display elements. Search behavior is also plastic, varying from a serial search of display and memory to parallel search independent of processing load (Schneider \& Shiffrin, 1977). This plasticity appears to depend on signal/ noise confusability and the consistency of mapping of forms to the target set.

Hoffman (1978) proposed a two-stage search model which suggested that the high speed of visual search is possible because the slow stages responsible for selective attention and memory comparison are guided by an initial parallel analysis of all display items.

The plasticity of search behavior is due to the subject's gradually relying on the parallel Stage 1 analysis when signal/noise ratio is high or when the same forms are consistently mapped to the target set. These are the conditions under which Schneider and Shiffrin (1977) propose that automatic detection develops.

The preceding experiments confirm several predictions of the two-stage search model. When the subject is in a varied mapping situation, detection accuracy benefits from decreasing the rate at which information is presented. Experiment 1 showed that this benefit is not attributable to variation in metacontrast between adjacent display characters. Experiment 2 suggested that slowing the presentation rate 
results in a faster transfer of the target from iconic to short-term memory, since the target evades an aftercoming visual mask sooner with sequential presentation.

Experiments 3 and 4 showed that the reason that transfer is faster with sequential presentation is that when several simultaneous inputs are present, a noise element will sometimes generate a higher Stage 1 similarity value than the target, resulting in a delay before the target is transferred. When noise elements are physically dissimilar from the target, no delay will occur and there should be no gain in accuracy or reduction in $\mathrm{RT}$ with sequential presentation. In addition, if circumstances permit, a decision may be made on the basis of Stage 1 analysis and both the effects of rate and memory set size will be eliminated. These predictions were confirmed, and, in addition, it was found that when the target was embedded in a homogeneous array of dissimilar characters, sequential presentation actually increased RT. This finding was attributed to a periodicity detection stage similar to the autocorrelation operation proposed by Uttal (1975). Thus, the transfer of display items to a serial comparison process can be controlled by an external means such as an indicator (Eriksen \& Collins, 1969), a noisy parallel comparison to the memory set items, or the periodic nature of the set of display characters that is not dependent on memory information.

These findings, together with the simulation model proposed by Hoffman (1978), provide support for the existence of a multistage, hierarchically organized visual information processing system. Thus, it is not possible to provide a simple answer to the questions raised in the introduction. Limitations in processing visual information may be due to memory comparison or selective attention. In some cases, no limitation will be apparent. The latter finding suggests that processing of visual forms to a high level can occur in parallel with no limitation in processing capacity. Attention may only be required when fine discriminations are necessary.

\section{REFERENCE NOTE}

1. Lappin, J. S. The relativity of choice behavior and the effect of prior knowledge on the speed and accuracy of recognition. Paper presented at the 1976 Conference on Mathematical and Theoretical Psychology, Bloomington, Indiana, April 1976.

\section{REFERENCES}

Anderson, J. A. A theory for the recognition of items from short memorized lists. Psychological Review, 1973, 80, 417-438.

Colegate, R. L., Hoffman, J. E., \& Eriksen, C. W. Selective encoding from multielement visual displays. Perception \& Psychophysics, 1973, 14, 217-224.
ERIKsen, C. W., \& Collins, J. F. Temporal course of selective attention. Journal of Experimental Psychology, 1969, 80, 254-261.

ERIKSEN, C. W., \& SPENCER, T. Rate of information processing in visual perception: Some results and methodological considerations. Journal of Experimental Psychology Monograph, 1969, 79, 1-16.

EsTES, W. K. Interactions of signal and background variables in visual processing. Perception \& Psychophysics, 1972, 12, 278-286.

GARDNER, G. T. Evidence for independent parallel channels in tachistoscopic perception. Cognitive Psychology, 1973, 4, 130-155.

Hoffman, J. E. Hierarchical stages in the processing of visual information. Perception \& Psychophysics, 1975, 18, 348-354.

Hoffman, J. E. Search through a sequentially presented visual display. Perception \& Psychophysics, 1978, 23, 1-11.

KINCHLA, R. A. Detecting target elements in multi-element arrays: A confusability model. Perception \& Psychophysics, 1974, 15, 149-158.

LAPPIN, J. S., \& BELL, H. H. The detection of coherence in moving random-dot patterns. Vision Research, 1975, 5, 1-8.

NeISSER, U. Cognitive psychology. New York: Appleton-CenturyCrofts, 1967.

SChNeIDER, W., \& ShIfFrin, R. M. Controlled and automatic human information processing: I. Detection, search, and attention. Psychological Review, 1977, 84, 1-66.

ShAw, M. L. Reaction time in reading a tachistoscopic display for a memory set item. Perception \& Psychophysics, 1977, 21, 15-25.

SHIFFrin, R. M., \& GaRDNER, G. T. Visual processing capacity and attentional control. Journal of Experimental Psychology, 1972, 93, 72-82.

SHIFFrin, R. M., \& GEISLER, W. S. Visual recognition in a theory of information processing. In R. Solso (Ed.), The Loyola symposium: Contemporary viewpoints in cognitive psychology. Washington: Winston, 1973.

SPENCER, T. J. Encoding time from ionic storage: A single-letter visual display. Journal of Experimental Psychology, 1971, 91, 18-24.

Sperting, G., Budiansky, J., Spivak, J., \& Johnson, M. C. Extremely rapid visual search: The maximum rate of scanning letters for the presence of a numeral. Science, 1971, 174, 307-311.

Sternberg, S., \& Scarborough, D. L. Parallel testing of stimuli in visual search. In Proceedings of the international symposium on visual information processing and control of motor activity. Sofia: Bulgarian Academy of Sciences, 1969.

UTTAL, W. R. An autocorrelation theory of form detection. Hillsdale, N.J: Eribaum, 1975.

WINER, B. J. Statistical principles in experimental design. New York: McGraw-Hill, 1962.

\section{NOTE}

1. The four-grouped condition might be expected to be intermediate between the four-spaced and eight-letter displays, since the target would receive interference on only one side when it appeared in the outer positions of the four-grouped display. Separate analysis of inner vs. outer positions was not required, since the two four-letter displays produced identical performance.

(Received for publication November 1, 1977; accepted February 10, 1978.) 